\title{
Population at high-risk of indoor heatstroke: the usage of cooling appliances among urban elderlies in Japan
}

\author{
Masahide Kondo $\cdot$ Masaji Ono $\cdot$ Kouichi Nakazawa $\cdot$ \\ Momoko Kayaba $\cdot$ Emiko Minakuchi · \\ Kazutoshi Sugimoto $\cdot$ Yasushi Honda
}

Received: 9 May 2012/ Accepted: 9 October 2012/Published online: 17 November 2012

(c) The Japanese Society for Hygiene 2012

\begin{abstract}
Objectives Heatstroke due to a heat wave during the summer is one of the commonly known health impacts of climate change in Japan. The elderly are particularly at high-risk of developing indoor heatstroke with poor prognosis. This study aims to describe the population among elderlies at high-risk of indoor heatstroke by focusing on the usage of cooling appliances.

Methods We conducted a web-based household survey in eight urban areas during the winter season of 2011. Households with a person aged 65 and over were selected as samples from panel members of a research firm, and the oldest member of the household was queried about his/her usage of cooling appliances. The population at high-risk of indoor heatstroke is defined as the elderly staying in a room without cooling appliances, or not using the installed
\end{abstract}

M. Kondo

Department of Health Care Policy and Health Economics,

Faculty of Medicine, University of Tsukuba, Tsukuba, Japan

M. Ono

Center for Environmental Health Sciences,

National Institute of Environmental Studies, Tsukuba, Japan

K. Nakazawa $\cdot$ M. Kayaba - E. Minakuchi $\cdot$ K. Sugimoto

Department of Health Care Policy and Management,

Graduate School of Comprehensive Human Sciences,

University of Tsukuba, Tsukuba, Japan

Y. Honda ( $\square)$

Department of Environmental Health,

Faculty of Health and Sport Sciences, University of Tsukuba,

Building D \#709, 1-1-1 Tennoudai, Tsukuba,

Ibaraki 305-8577, Japan

e-mail: honda@taiiku.tsukuba.ac.jp cooling appliances, or turning the cooling appliances on only when the room temperature is above $28^{\circ} \mathrm{C}$.

Results 15.4 and $19.1 \%$ of the elderlies living in urban areas of Japan are identified as at high-risk of indoor heatstroke during activity time and sleeping time, respectively, according to the definition of high-risk of indoor heatstroke in this study.

Conclusions These figures are not negligible since the consequences of heatstroke are grave, but its risk can be eliminated by an appropriate usage of cooling appliances. The preventive interventions are needed to protect the elderlies at high-risk of heatstroke.

Keywords Climate change Cooling appliance · Elderly · Global warming · Heatstroke

\section{Introduction}

The warming air temperature observed in the global climate change is progressing at an unprecedented rate, and development of adaptation measures beyond the mere evaluation of impacts has become an urgent issue [1]. Heatstroke due to severe heat wave in the summer has been one of the most obvious health impacts of global warming in Japan. The elderly are particularly at high-risk of developing indoor heatstroke with poor prognosis that can be fatal [2, 3], compared to younger people. However, it is possible to eliminate the risk of indoor heatstroke, if the elderly appropriately use the cooling appliances such as an electric fan or an air conditioner. The purpose of this study is to describe the population at high-risk (PAHR) of indoor heatstroke among elderlies in order to develop an urban community-based prevention programme. 


\section{Materials and methods}

We conducted a web-based questionnaire survey in February of 2011, before the Great East Japan Earthquake, employing a research firm, which has more than 1.5 million panel members nationwide. Eight urban areas were chosen as prospective fields for our development of an urban community-based heatstroke prevention programme, which will follow this baseline survey. The chosen areas from north to south are: Sapporo, Sendai, Saitama, Tokyo's 23 wards, Chiba, Kobe, Kitakyushu and Nagasaki. The latitude, average year value of mean air temperature in August, average year value of days exceeding $30^{\circ} \mathrm{C}$ and average year value of mean relative humidity in August of each area are shown in Table 1. These average values are 'mean values' disseminated by the Japan Meteorological Agency, which are calculated from 1981 to 2010 values. Households with a person aged 65 and over were selected as samples from panel members of the research firm, and the usage of cooling appliances during the summer by the oldest member in the household was queried. Surrogate reporting was allowed, if the target elderly had difficulty in self-reporting via the Internet. Rooms where the elderly spend the longest time during the daytime and nighttime were asked about, and those were defined as a sitting room, a bedroom, or a sitting cum bedroom. Questions included the installation of cooling appliances in those rooms and their usage patterns. Four usage patterns during daytime were given to the respondents as alternatives: 'constant use', 'only when hot', 'other' and 'never use'. For those who chose 'only when hot', room air temperature at which they turned on appliances was asked about, and they were allowed to report the temperature intuitively. Similarly, four usage patterns during nighttime were also given to the respondents: 'constant use', 'set timer', 'other' and 'never use'. Sleeping was assumed and explained for the respondents in this query, so that room air temperature at which they turned on appliances was not asked. An elderly was defined as at high-risk of indoor heatstroke if he/she stays in a room with no cooling appliance, or never uses installed cooling appliances. Additionally, an elderly using cooling appliances was also defined as at high-risk, if he/she turns the appliances on only when the room temperature is $28{ }^{\circ} \mathrm{C}$ or above during activity time. The threshold, $28{ }^{\circ} \mathrm{C}$, was set according to the heat stress standard ISO 7243 [4]. Bias due to surrogate reporting were also appraised in order to examine any unanticipated systematic effect on responses, because there was a concern whether the Internet using family member was able to report an everyday behaviour of the older family member such as the usage of cooling appliances. If substantial differences were found between surrogate reporting and self-reporting, the results would be less certain than situations without such differences.

Table 1 Area descriptions and backgrounds of elderlies in each household

\begin{tabular}{|c|c|c|c|c|c|c|c|c|c|}
\hline & Sapporo & Sendai & Saitama & $\begin{array}{l}\text { Tokyo's } \\
23 \text { wards }\end{array}$ & Chiba & Kobe & Kitakyushu & Nagasaki & All \\
\hline Latitude of $\operatorname{area}^{\mathrm{a}}\left({ }^{\circ} \mathrm{N}\right)$ & 43.03 & 38.16 & 35.53 & 35.41 & 35.36 & 34.42 & 33.51 & 32.44 & \\
\hline $\begin{array}{l}\text { Average year value of mean air } \\
\text { temperature in August }{ }^{\mathrm{a}}\left({ }^{\circ} \mathrm{C}\right)\end{array}$ & 22.3 & 24.2 & 26.6 & 27.4 & 26.7 & 28.3 & 27.4 & 27.9 & \\
\hline $\begin{array}{l}\text { Average year value of days } \\
\text { with temperatures exceeding } \\
30{ }^{\circ} \mathrm{C}^{\mathrm{a}} \text { (days) }\end{array}$ & 8.0 & 17.9 & 52.9 & 48.5 & 42.3 & 54.9 & 54.5 & 54.2 & \\
\hline $\begin{array}{l}\text { Average year value of mean } \\
\text { relative humidity in August }{ }^{\mathrm{a}}(\%)\end{array}$ & 75 & 81 & N.A. & 71 & 78 & 71 & N.A. & 75 & \\
\hline Number of responses $(n)$ & 763 & 410 & 488 & 3,386 & 363 & 663 & 258 & 125 & 6,456 \\
\hline \multicolumn{10}{|l|}{ Sex } \\
\hline Male $(\%)$ & 47.4 & 55.6 & 56.4 & $52.6^{*}$ & $61.2 *$ & $55.8 *$ & 53.1 & 43.2 & $53.1 *$ \\
\hline \multicolumn{10}{|l|}{ Age } \\
\hline Average [years, (SD)] & $\begin{array}{l}74.2^{*} \\
(7.2)\end{array}$ & $\begin{array}{l}74.8^{*} \\
(7.4)\end{array}$ & $\begin{array}{l}74.2^{*} \\
(7.8)\end{array}$ & $\begin{array}{l}75.0^{*} \\
(7.4)\end{array}$ & $\begin{array}{r}73.3^{*} \\
(7.0)\end{array}$ & $\begin{array}{l}74.3^{*} \\
(7.4)\end{array}$ & $\begin{array}{r}74.6^{*} \\
(7.4)\end{array}$ & $\begin{array}{l}75.8^{*} \\
(7.5)\end{array}$ & $\begin{array}{r}74.7 * \\
(7.4)\end{array}$ \\
\hline \multicolumn{10}{|l|}{ Respondent } \\
\hline Surrogate reporting $(\%)$ & 77.1 & 82.2 & 73.4 & 77.8 & 65.8 & 70.1 & 78.7 & 80.8 & 76.3 \\
\hline \multicolumn{10}{|l|}{ Room } \\
\hline Sitting cum bedroom $(\%)^{\mathrm{b}}$ & $19.1 *$ & 20.0 & 26.0 & $30.5^{*}$ & $24.0 *$ & $31.2 *$ & $31.4^{*}$ & 25.6 & $27.8 *$ \\
\hline
\end{tabular}

* Statistically significant $(p<0.05)$ difference between self-reporting and surrogate reporting by $\chi^{2}$ test/Fisher's exact test for $2 \times 2$ table or $\mathrm{t}$ test for means

a Adopted from Japan Meteorological Agency

b Separate rooms for sitting room and bedroom, otherwise 
The research ethics committee of Faculty of Health and Sport Sciences, University of Tsukuba, agreed to the conduct of this survey.

\section{Results}

Collected responses from 6,456 households are analysed as in the following. Since the quota sampling method was applied for contracted panel members of a research firm, the conventional response rate is not calculable. Table 1 summarises the backgrounds of target elderlies in each household. It is seen that $53.1 \%$ of all target elderlies are male, and their average age is 74.7 years old. Also, $27.8 \%$ spend most of their time in their sitting cum bedroom, while the rest use separate rooms for sitting room and bedroom. And $76.3 \%$ of the responses are by surrogate reporting. Between self-reporting and surrogate reporting, differences in their average age are consistently found to be statistically significant, which may be explained by the difference in affinity to the Internet by age. The older the target elderlies, the more the surrogate reporting, which would reflect a fact that the older uses the Internet less than the younger. Differences by surrogate reporting are also suggested in the target elderlies' sex and the room in which they stay.

Table 2 shows the installation of cooling appliances. In all eight areas, 54.4 and $78.2 \%$ of sitting rooms are equipped with an electric fan and air conditioner, respectively, while $6.0 \%$ have no cooling appliance. For the bedrooms, 45.8 and $61.7 \%$ are equipped with an electric fan and air conditioner, respectively, while $15.6 \%$ have no cooling appliance. And 65.6 and $78.1 \%$ of sitting cum bedrooms are equipped with electric fan and air conditioner, respectively, while $5.0 \%$ have no cooling appliance. Indifferent figures are found in six areas, Saitama and five areas south of Saitama. The diffusion of air conditioners is notably low in northern areas, Sapporo and Sendai, compared to the other areas. Proportions of rooms without any cooling appliance are remarkably large in Sapporo, 27.6-58.0\%; and also large in Sendai, 6.1-20.4\%. These can be naturally explained by the cooler and shorter summers in these areas where improving coolness inside rooms is not so necessary. No systematic differences are found between self-reporting and surrogate reporting, although several statistically significant differences are found. In particular, figures of "no cooling appliance" are consistently found to be statistically insignificant.

Table 3 shows the usage of cooling appliances where installed. During activity time, the most common usage is turning the appliances on when one feels a room too hot, regardless of the type of appliance, either an electric fan or an air conditioner. In all areas, 76.4 and $73.9 \%$ of the elderlies use an electric fan and air conditioner only when they feel hot, respectively. And 19.2 and $21.7 \%$ use an electric fan and air conditioner constantly throughout their activity time, respectively, while 2.6 and $3.4 \%$ never use them, respectively. Indifferent figures are found in six areas, Saitama and other areas south of Saitama. Proportions of "constant use" throughout their activity time are remarkably small in two northern areas, Sapporo and Sendai. These are probably due to the cooler and shorter summers in these areas compared to the rest. During sleeping time, the most common usage is setting up an on/ off timer, regardless of the type of appliance. In all areas, 65.9 and $67.8 \%$ of the elderlies use an electric fan and air conditioner with timer setting, respectively. And 12.8 and

Table 2 Installation of cooling appliances

\begin{tabular}{|c|c|c|c|c|c|c|c|c|c|}
\hline & Sapporo & Sendai & Saitama & $\begin{array}{l}\text { Tokyo's } \\
23 \text { wards }\end{array}$ & Chiba & Kobe & Kitakyushu & Nagasaki & All \\
\hline \multicolumn{10}{|l|}{ Sitting room } \\
\hline Electric fan $(\%)$ & 62.1 & 53.7 & 51.7 & $55.4 *$ & $53.6^{*}$ & 59.9 & 49.2 & $55.9 *$ & $54.4 *$ \\
\hline Air conditioner $(\%)$ & 18.0 & 73.5 & $88.7 *$ & $90.6 *$ & 89.9 & 86.6 & 87.0 & 88.2 & $78.2^{*}$ \\
\hline No cooling appliance (\%) & 27.6 & 7.0 & 2.4 & 1.7 & 2.9 & 2.0 & 2.3 & 2.2 & 6.0 \\
\hline \multicolumn{10}{|l|}{ Bedroom } \\
\hline Electric fan $(\%)$ & 39.7 & $54.3 *$ & $42.7 *$ & $47.9 *$ & 53.3 & $54.2 *$ & $53.1 *$ & 50.5 & $45.8^{*}$ \\
\hline Air conditioner $(\%)$ & 3.4 & 36.3 & 76.7 & 72.3 & 64.9 & 69.5 & 63.3 & 64.5 & $61.7 *$ \\
\hline No cooling appliance (\%) & 58.0 & 20.4 & 7.6 & 7.5 & 8.7 & 10.7 & 9.0 & 9.7 & 15.6 \\
\hline \multicolumn{10}{|l|}{ Sitting cum bedroom } \\
\hline Electric fan $(\%)$ & 63.7 & 69.5 & 62.2 & 70.9 & 63.2 & 73.4 & $71.6^{*}$ & 93.8 & 65.6 \\
\hline Air conditioner $(\%)$ & 10.3 & 61.0 & 87.4 & 89.8 & 74.7 & 79.7 & 81.5 & 78.1 & 78.1 \\
\hline No cooling appliance (\%) & 32.2 & 6.1 & 2.4 & 1.6 & 4.6 & 2.4 & 1.2 & 0.0 & 5.0 \\
\hline
\end{tabular}

* Statistically significant $(p<0.05)$ difference between self-reporting and surrogate reporting by $\chi^{2}$ test/Fisher's exact test for $2 \times 2$ table 
Table 3 Usage of cooling appliance
* Statistically significant $(p<0.05)$ difference between self-reporting and surrogate reporting by $\chi^{2}$ test/Fisher's exact test for $2 \times 4$ table. Long square brackets indicate the sum columns of $2 \times 4$ tables tested

\begin{tabular}{|c|c|c|c|c|c|c|c|c|c|}
\hline & Sapporo & Sendai & Saitama & Tokyo's 23 wards & Chiba & Kobe & Kitakyushu & Nagasaki & All \\
\hline \multicolumn{10}{|l|}{ Activity time } \\
\hline \multicolumn{10}{|l|}{ Electric fan } \\
\hline Constant use (\%) & 4.0 & $12.97^{*}$ & $26.2]^{*}$ & $21.7]^{*}$ & $20.27^{*}$ & $22.4]^{*}$ & 24.1 & 17.1 & $19.27^{*}$ \\
\hline Only when hot (\%) & 90.8 & 82.8 & 71.4 & 73.9 & 76.8 & 72.2 & 70.3 & 80.5 & 76.4 \\
\hline Other $(\%)$ & 1.1 & 3.0 & 1.4 & 1.8 & 2.0 & 2.4 & 1.4 & 1.2 & 1.8 \\
\hline Never use (\%) & 4.2 & $1.3\rfloor$ & $1.0]$ & $2.6]$ & $1.0\rfloor$ & $3.1\rfloor$ & 4.1 & 1.2 & $2.6]$ \\
\hline \multicolumn{10}{|l|}{ Air conditioner } \\
\hline Constant use (\%) & 5.6 & 13.7 & 21.3 & $24.8]^{*}$ & $16.0]^{*}$ & 17.1 & 23.2 & 15.9 & $21.77^{*}$ \\
\hline Only when hot (\%) & 89.7 & 78.7 & 75.1 & 71.0 & 80.2 & 77.7 & 73.2 & 80.4 & 73.9 \\
\hline Other $(\%)$ & 1.6 & 1.0 & 0.9 & 1.1 & 0.6 & 0.9 & 0.5 & 0.9 & 1.0 \\
\hline Never use (\%) & 3.2 & 6.5 & 2.7 & $3.0\rfloor$ & $3.2\rfloor$ & 4.3 & 3.2 & 2.8 & $3.4\rfloor$ \\
\hline \multicolumn{10}{|l|}{ Sleeping time } \\
\hline \multicolumn{10}{|l|}{ Electric fan } \\
\hline Constant use (\%) & $3.0]^{*}$ & 6.4 & 14.8 & $14.3]^{*}$ & 12.4 & 15.5 & 17.8 & 11.7 & $12.8]^{*}$ \\
\hline Set timer $(\%)$ & 67.8 & 72.8 & 68.8 & 63.7 & 70.3 & 63.9 & 67.8 & 70.1 & 65.9 \\
\hline Other $(\%)$ & 6.8 & 6.0 & 4.2 & 5.2 & 4.5 & 6.8 & 4.6 & 2.6 & 5.4 \\
\hline Never use (\%) & $22.5\rfloor$ & 14.9 & 12.2 & $16.8]$ & 12.9 & 13.8 & 9.9 & 15.6 & $15.9]$ \\
\hline \multicolumn{10}{|l|}{ Air conditioner } \\
\hline Constant use (\%) & 13.9 & 8.9 & 9.9 & 14.7 & 8.6 & 12.9 & 14.6 & 7.1 & 13.3 \\
\hline Set timer $(\%)$ & 61.1 & 65.7 & 74.1 & 66.8 & 67.2 & 68.5 & 68.0 & 76.5 & 67.8 \\
\hline Other (\%) & 11.1 & 8.3 & 5.3 & 5.6 & 7.8 & 3.5 & 3.9 & 5.9 & 5.5 \\
\hline Never use (\%) & 13.9 & 17.2 & 10.7 & 13.0 & 16.4 & 15.1 & 13.5 & 10.6 & 13.4 \\
\hline
\end{tabular}

$13.3 \%$ keep an electric fan and air conditioner running during their sleeping time, respectively, while 15.9 and $13.4 \%$ never use them while sleeping, respectively. Regarding the usage of electric fans, Sapporo and Sendai, are different from the other areas such that proportions of "constant use" are small and that those of "never use" are large. These can also be explained by the cooler and shorter summers in these areas. Regarding the usage of air conditioners, indifferent figures are found in all eight areas. It is noteworthy that figures of the usage of air conditioners during sleeping time are consistently not statistically significant between self-reporting and surrogate reporting.

Figure 1 shows the room temperature when the cooling appliances are turned on during activity time by those who are in rooms equipped with an electric fan and/or air conditioner, and use them only when they feel too hot. In all areas, 19.0 and $26.8 \%$ of them do not turn on their electric fan and air conditioner, respectively, even when the room temperature is $28{ }^{\circ} \mathrm{C}$ or above. Proportions of those who turn on their electric fan when the room temperature is $28{ }^{\circ} \mathrm{C}$ or above range from $3.5 \%$ in Nagasaki to $22.0 \%$ in Kitakyushu, while proportions for "air conditioner" range from $22.3 \%$ in Sapporo to
$31.3 \%$ in Chiba. Except the smallest figure, $3.5 \%$ for "electric fan" in Nagasaki, indifferent figures are found in all areas irrespective of "electric fan" or "air conditioner". The effect of surrogate reporting is implied, since statistically significant differences between self-reporting and surrogate reporting are found in the majority of cumulative utilisation curves.

Table 4 summarises identified elderly's PAHR of indoor heatstroke. In all areas, 15.4 and $19.1 \%$ of the elderlies fall in the category of at high-risk defined in this study during activity time and sleeping time, respectively. The PAHR during activity time are those who stay in a room without any cooling appliance, $5.7 \%$; those who never use installed cooling appliances, $1.6 \%$; and those who turn the appliances on only when the room temperature exceeds $28{ }^{\circ} \mathrm{C}, 8.2 \%$; while PAHR during sleeping time are those who stay in a room without any cooling appliance, $12.7 \%$; and those who never use installed cooling appliances during their sleep, $6.4 \%$. In light of the motivation of this study, the development of an urban community-based prevention programme, areal characteristics instead of national averages are of interest. Two northern areas, Sapporo and Sendai, are different from the rest as already 
Cumulative utilisation $\%$

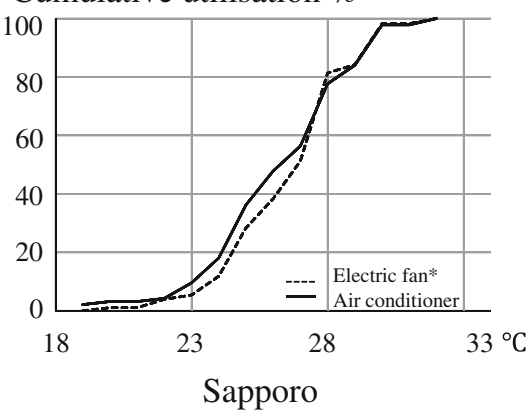

Cumulative utilisation $\%$

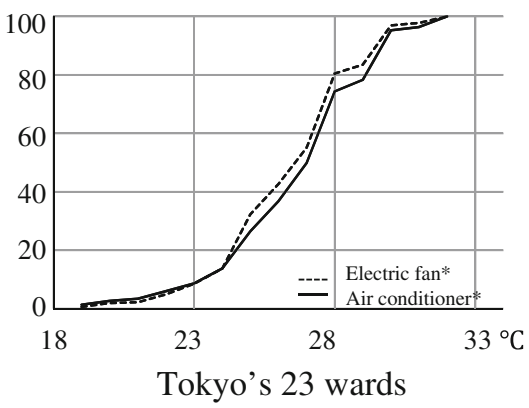

Cumulative utilisation $\%$

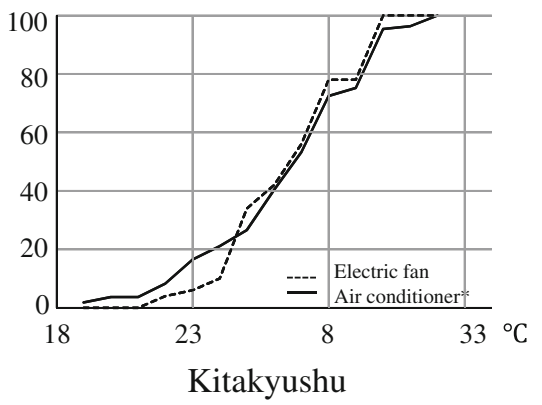

Cumulative utilisation $\%$

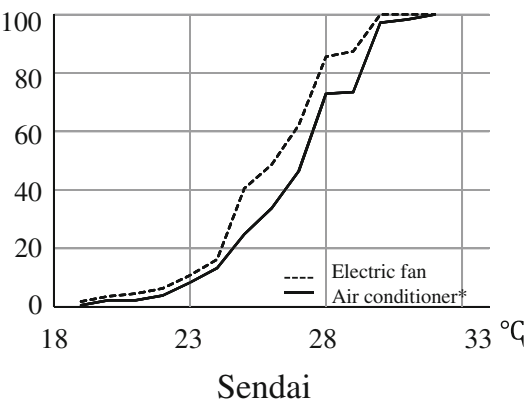

Cumulative utilisation $\%$

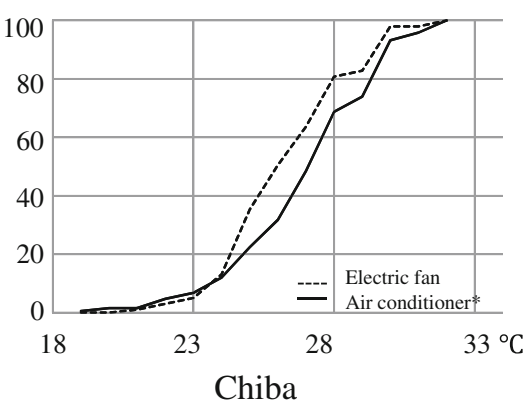

Cumulative utilisation $\%$

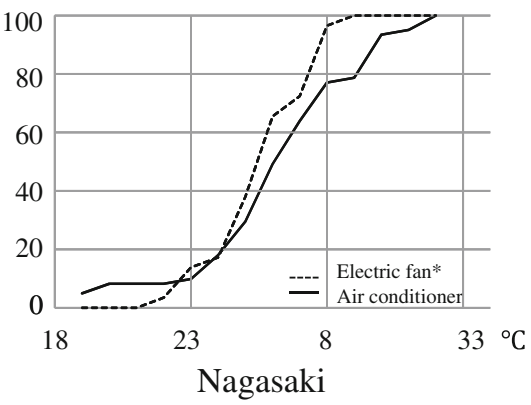

Cumulative utilisation $\%$

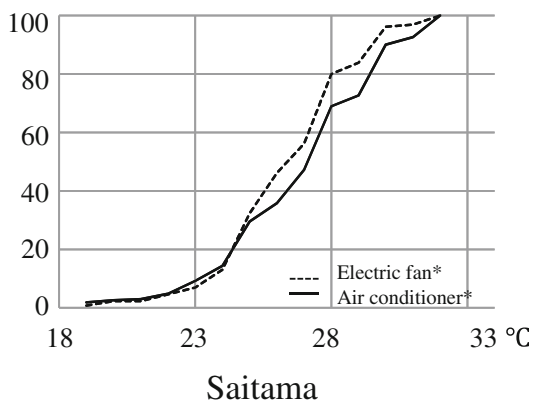

Cumulative utilisation $\%$

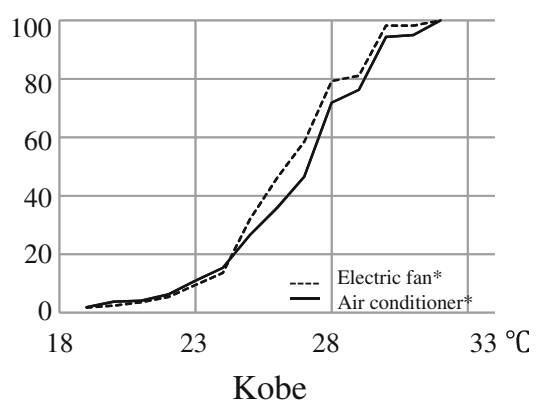

Cumulative utilisation \%

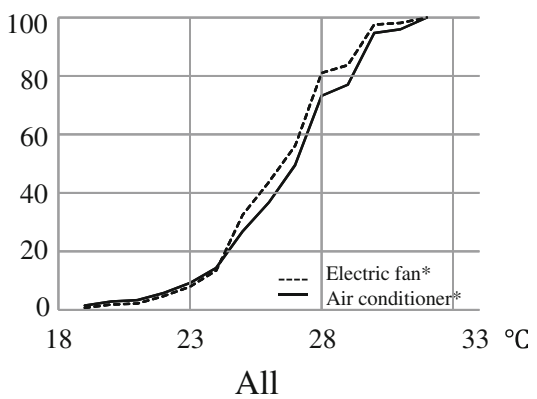

Fig. 1 Room temperature to turn on the cooling appliances during activity time. *Statistically significant $(p<0.05)$ difference between selfreporting and surrogate reporting by the Mann-Whitney test

found in Tables 2 and 3. Their large PAHR owes to large figures of "no installation" of cooling appliances. No systematic differences are found between self-reporting and surrogate reporting here, although a few statistically significant differences are found in "activity time".

\section{Discussion}

There were 15.4 and $19.1 \%$ of the elderlies living in the urban areas of Japan as identified as PAHR of indoor heatstroke during activity time and sleeping time, respectively, according to the definition of PAHR of indoor heatstroke in this study. These figures are not negligible since heatstroke consequences may be fatal, while the risk is avoidable through appropriate usage of cooling appliances. The development of prevention programmes is now necessary and crucial [5]. Between their activity time and sleeping time, it is understandable that elderlies tend to feel more reluctant to use cooling appliances while sleeping, but heat stress exposure during sleeping time is an important risk factor [6].

Larger PAHR are found in two northern areas, Sapporo and Sendai compared to another six areas, Saitama and other areas south of Saitama, due to large proportions of those who stay in rooms without cooling appliances. It is essential to note that these figures only reflect the usage of cooling appliances, and do not reflect the exposure to heat stress inside the room. Cooler and shorter summer days in northern areas probably result in less heat stress inside the 
Table 4 Populations at high-risk (PAHR) of indoor heatstroke among elderlies

\begin{tabular}{|c|c|c|c|c|c|c|c|c|c|}
\hline & Sapporo & Sendai & Saitama & Tokyo's 23 wards & Chiba & Kobe & Kitakyushu & Nagasaki & All \\
\hline \multicolumn{10}{|l|}{ Activity time } \\
\hline No installation (\%) & 28.4 & 6.8 & 1.6 & $2.4]^{*}$ & $3.37^{*}$ & 2.1 & 1.9 & $1.6]^{*}$ & $5.7]^{*}$ \\
\hline Never use $(\%)$ & 2.5 & 2.2 & 0.6 & 1.3 & 1.4 & 2.0 & 1.9 & 1.6 & 1.6 \\
\hline Turn on only when room temperature is above $28{ }^{\circ} \mathrm{C}(\%)$ & 8.4 & 7.1 & 9.0 & $8.0\rfloor$ & $9.9]$ & 8.9 & 8.5 & $4.0]$ & $8.2]$ \\
\hline Total $(\%)$ & 39.3 & 16.1 & 11.3 & 11.7 & 14.6 & 13.0 & 12.4 & 7.2 & 15.4 \\
\hline \multicolumn{10}{|l|}{ Sleeping time } \\
\hline No installation (\%) & 53.1 & 17.6 & 5.9 & 6.0 & 7.7 & 8.1 & 6.6 & 7.2 & 12.7 \\
\hline Never use (\%) & 7.9 & 7.1 & 4.5 & 6.4 & 6.1 & 6.8 & 4.7 & 4.8 & 6.4 \\
\hline Total (\%) & 60.9 & 24.6 & 10.5 & 12.4 & 13.8 & 14.9 & 11.2 & 12.0 & 19.1 \\
\hline
\end{tabular}

* Statistically significant $(p<0.05)$ difference between self-reporting and surrogate reporting by $\chi^{2}$ test/Fisher's exact test for $2 \times 3 / 2 \times 2$ table. Long square brackets indicate the sum columns of $2 \times 3$ tables tested

room, which lead to less incidence of indoor heatstroke among the elderlies in these areas.

The definition of PAHR of indoor heatstroke in this study, especially the use of the cut-off value of $28^{\circ} \mathrm{C}$ for turning on the cooling appliances during activity time, needs to be scrutinised. It is known that there is a risk of indoor heatstroke even when the room temperature is below $28{ }^{\circ} \mathrm{C}$ [4]. Then the threshold of $28{ }^{\circ} \mathrm{C}$ applied in this study implies that the results of the "activity time" are underestimated. Also, northern areas are known to have less than $28{ }^{\circ} \mathrm{C}$ optimum temperatures, which is defined as a daily maximum air temperature with the lowest mortality rate based on a V-shaped relationship between daily maximum air temperature and mortality [7]. This implies a need for preventive interventions even when the air temperature is below $28^{\circ} \mathrm{C}$, although these air temperatures refer to outside air temperatures and not inside.

We had a concern about bias due to surrogate reporting, especially regarding the room temperature at which the cooling appliances were turned on. This bias can happen in cases of daily trivial behaviour. Considering that the elderly's thermal sensitivity is lower compared to the young [8, 9], the bias should be such that the surrogate reporting may result in a lower temperature. However, in the summary table of PAHR of indoor heat stroke (Table 4), no such tendency about the temperature was found. Nevertheless, we think that results with a statistically significant difference between surrogate reporting and self-reporting are less certain than those without statistical significance, and consider that this is a limitation of our survey data.

Sampling bias owing to the use of a web-based questionnaire survey employing a research firm limits the accuracy of the size of PAHR. There should be a difference between the panel member households and the whole households as the sampling frame. Nevertheless, we consider that the results elucidate room for developing prevention programmes. Our sample households being the panel members of the IT research firm are more likely to have cooling appliances in their rooms compared to the households nationwide, since they are considered to be well informed about electric appliances. If this is the case, our results would be an underestimation.

This study took place just before the Great East Japan Earthquake, which was followed by the electricity savings in damaged areas like Sendai, Saitama, Tokyo's 23 wards and Chiba. Advocated policy heavily covered by the media has surely made the elderly refrain from using cooling appliances, which put a larger PAHR. We think appropriate protection and information or education against heat stress is necessary for the elderly even in the context of electricity shortage.

Two types of preventive interventions are implied from this study. For elderlies staying in rooms with cooling appliances, behaviour modification such as turning the appliances on even when the room temperature is below $28{ }^{\circ} \mathrm{C}$ is imperative. In doing so, risk communication and health education is probably not enough, and provision of devices to prompt the use of appliances like an alarm according to the Wet-Bulb Globe Temperature (WBGT) or a thermo-hygrometer with instructions to keep the room lower than $28^{\circ} \mathrm{C} / 60 \%$ could be beneficial [10]. For elderlies staying in rooms without any cooling appliance, providing subsidy for installing cooling appliances could be justified in light of the outbreak of indoor heatstroke among the elderly $[2,10]$. It is certain that areal characteristics need to be considered when designing operational interventions. Our results suggest that northern areas have different PAHR elderlies compared to the rest. "No installation" of cooling appliances is large, which implies 
the importance of providing subsidy for installing cooling appliances. However, taking less indoor heat stress during the summer in these areas into account, such subsidy could be less effective than in the other areas, since people have less demand for cooling appliances in the first place. The provision of a cooling shelter using community centres, for example, might work, which would be less costly. Further studies on wider factors related to indoor heatstroke such as incidence or lifestyle are particularly required for developing effective intervention in these areas.

Acknowledgments This study was funded by the Environment Research and Technology Development Fund S-8 from Ministry of the Environment, Japan.

Conflict of interest The authors declare there are no conflict of interests.

\section{References}

1. Intergovernmental Panel on Climate Change. Climate Change 2007-impacts, adaptation and vulnerability: Working Group II contribution to the Fourth Assessment Report of the IPCC. Cambridge: Cambridge University Press; 2008.
2. Kondo M, Honda Y, Ono M. Growing concern about heatstroke this summer in Japan after Fukushima nuclear disaster. Environ Health Prev Med. 2011;16:279-80.

3. Lin YK, Ho TJ, Wang YC. Mortality risk associated with temperature and prolonged temperature extremes in elderly populations in Taiwan. Environ Res. 2011;111:1156-63.

4. Parsons K. Heat stress standard ISO 7243 and its global application. Ind Health. 2006;44:368-79.

5. Martinez GS, Imai C, Masumo K. Local heat stroke prevention plans in Japan: characteristics and elements for public health adaptation to climate change. Int J Environ Res Public Health. 2011;8:4563-81.

6. Vandentorren S, Bretin P, Zeghnoun A, Mandereau-Bruno L, Croisier A, Cochet C, et al. August 2003 heat wave in France: risk factors for death of elderly people living at home. Eur $\mathrm{J}$ Public Health. 2006;16:583-91.

7. Honda Y, Kabuto M, Ono M, Uchiyama I. Determination of optimum temperature using climate data. Environ Health Prev Med. 2007;12:209-16.

8. Guergova S, Dufour A. Thermal sensitivity in the elderly: a review. Ageing Res Rev. 2011;10:80-92.

9. Inoue Y. Prevention of heat illness in children and the elderly. Jpn J Biometeorol. 2004;41:61-6. (article in Japanese).

10. Miyake Y, Aruga T, Inoue K, Okudera H, Kitahara T, Shimazaki $\mathrm{S}$, et al. Characteristics of heatstroke patients in Japan; Heatstroke STUDY2008. JAAM. 2010;21:230-44. (article in Japanese). 